\title{
Intestinal Assimilation of a Tetrapeptide in the Rat
}

\author{
OBLIGATE FUNCTION OF BRUSH BORDER AMINOPEPTIDASE
}

\author{
Kenneth W. Smithson and Gary M. Gray \\ From the Division of Gastroenterology, Department of Medicine, Stanford University School of \\ Medicine, Stanford, California 94305
}

A в S T R A C T The small intestine is capable of taking up peptide nutrients of two or three amino acid residues, but the mechanism of intestinal assimilation of larger oligopeptides has not been established. The amino-oligopeptidase of the intestinal brush border possesses high specificity for oligopeptides having bulky side chains and is a candidate for a crucial role in the overall assimilation of dietary protein.

Rat jejunum was used for in vitro gut sac and in vivo perfusion experiments with Gly-L-Leu-Gly-Gly $(2 \mathrm{mM})$ as the test substrate with analysis of parent peptide and products by automatic ion-exchange chromatography. In these experiments, the tetrapeptide disappeared rapidly from the test solution (20 $\mu \mathrm{mol} / \mathrm{s}$ per $\mathrm{cm}^{2}$ in vitro; $17 \mu \mathrm{mol} / \mathrm{s}$ per $\mathrm{cm}^{2}$ in vivo) by sequential removal of amino acid residues from the $\mathrm{N}$-terminus to yield amino acids and the C-terminal dipeptide. In gut sac experiments, $61-100 \%$ of these products of hydrolysis appeared in the incubation medium and the remainder in the tissue. In contrast, only small amounts of hydrolytic products were found within intestinal lumen in vivo.

Gly-L-Pro $(10 \mathrm{mM})$, a peptide known to be transported intact but not to be hydrolyzed by the brush border aminopeptidase, failed to inhibit Gly-L-LeuGly-Gly disappearance suggesting that the tetrapeptide does not utilize the known intact transport mechanism.

Hypoxic conditions $\left(\mathrm{N}_{2}\right.$ atmosphere) in vitro markedly inhibited transport of glucose, leucine, and

This work was presented in part at the Annual Meeting of the American Federation for Clinical Research-American Society for Clinical Investigation, Atlantic City, N. J., 4 May 1975, and published as an abstract: 1975. Clin. Res. 23: $257 \mathrm{~A}$.

Dr. Smithson is a trainee under U. S. Public Health Service National Institutes of Health Training Grant in Gastroenterology AM 05418. Dr. Gray is recipient of U. S. Public Health Service National Institutes of Health Research Career Development Award AM 47443.

Received for publication 13 January 1977 and in revised form 31 March 1977.
Gly-Gly but failed to impair Gly-L-Leu-Gly-Gly disappearance suggesting that the first step in assimilation of the tetrapeptide does not involve a transport process.

Disappearance of the tetrapeptide was completely blocked by L-leucyl- $\beta$-naphthylamide $(10 \mathrm{mM})$, a specific substrate for brush border aminopeptidase and by the phthalimido derivative of L-leucine bromomethyl ketone, a potent peptidase inhibitor. Hence, the amino-oligopeptidase at the intestinal surface appears to be essential for the initial stages of assimilation of this model tetrapeptide.

\section{INTRODUCTION}

Pancreatic proteases within the intestinal lumen act on dietary protein to produce a mixture composed of oligopeptides of two to six amino acid residues and a lesser amount of free amino acids. Since nearly all peptide nutrients appear to enter the portal circulation in the form of free amino acids (1), the intestinal mucosa appears to be responsible for their final degradation. Many dipeptides appear to be assimilated by a specific mechanism at more rapid rates than occur for an equivalent mixture of the component amino acids $(2,3)$. Further, the fact that patients with congenital defects in intestinal amino acid transport are capable of assimilating peptides containing the relevant amino acids attests to the physiological importance of this peptide transport system (4). Thus, the concept of transport of intact dipeptides by mammalian small intestine has gained wide acceptance and this process is presumably complemented by intracellular hydrolysis via cytosol peptidases (5).

The fate of a peptide containing three or more residues may depend upon its amino acid composition. For instance, the homopeptide Gly-Gly-Gly consists of an amino acid having only a hydrogen atom as its side chain $(0.3 \AA)$, and there is strong 
evidence that it is assimilated intact by the peptide transport mechanism $(6,7)$. In contrast, Leu-Leu-Leu is much more bulky because of the long hydrocarbon side chain $(3.6 \AA)$ of each leucine residue. Intestinal assimilation of this peptide is associated with appreciable intraluminal accumulation of free leucine suggesting that it may be primarily hydrolyzed rather than transported intact (7). Aminopeptidases are present in the brush border membrane of mammalian small intestine at specific activities comparable to those found for the disaccharidases (8), the carbohydrases known to be essential for surface hydrolysis of dietary carbohydrate. These membrane enzymes possess maximal specificity for oligopeptides having three or more amino acid residues with bulky side chains (9), but their role in protein assimilation has not been established.

In this paper, we have used specific inhibitors of both peptide transport and of the amino-oligopeptidases to determine the mechanism of intestinal assimilation of a mixed peptide, Gly-Leu-Gly-Gly. The results indicate that the tetrapeptide, rather than being transported intact, is hydrolyzed on the intestinal cell surface to its constituent amino acids and C-terminal dipeptide which are subsequently transported by known mechanisms. Hence the brush border amino-oligopeptidase appears to be essential for the initial stage of digestion of this model oligopeptide.

\section{METHODS}

Materials. Dipeptides, glucose oxidase, leucyl- $\beta$ napthylamide and amino acids ${ }^{1}$ were obtained from Sigma Chemical Co. (St. Louis, Mo.) and Gly-Leu-Gly-Gly ${ }^{2}$ and Leu-Gly-Gly were from Cyclo Chemicals Corp., Miami, Fla.). All were found pure by ion-exchange and thin-layer chromatography (10). $p$-(OH)-mercuribenzoate was obtained from Eastman Organic Chemicals Div., Eastman Kodak Co. (Rochester, N. Y.). The phthalmido derivative of leucine bromomethyl ketone (LPK) ${ }^{3}$ was a gift of Dr. Gordon Prescott and Dr. George Glover. Glycine, leucine, Gly-Gly, inulin, and glucose were uniformly labeled ${ }^{14} \mathrm{C}$-products from New England Nuclear (Boston, Mass.) and were made pure by thinlayer chromatography. All other chemicals were of reagent grade.

Unless otherwise stated, Ringer's solution, pH 7.4 (buffer A), was used for all experiments; it contained $140 \mathrm{mM}$

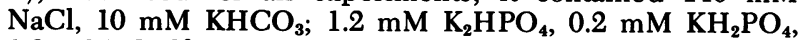
$1.2 \mathrm{mM} \mathrm{CaCl}, 1.2 \mathrm{mM} \mathrm{MgCl}$, and $\left[{ }^{4} \mathrm{C}\right]$ inulin as a nonabsorbable extracellular marker.

Preparation of everted gut sacs. Male Sprague-Dawley

${ }^{1}$ All amino acids, whether free or as components of peptides, were in the naturally occurring $\mathrm{L}$-form. The designation is omitted in this paper.

2 The conventional three-letter abbreviation for amino acids in peptides is used in this paper.

${ }^{3}$ Abbreviations used in this paper: LNA, leucyl- $\beta$ naphthylamide; LPK, phthalimido derivative of leucine bromomethyl ketone. rats, $250-300 \mathrm{~g}$, were allowed access to Berkeley lab chow (Berkeley Scientific Laboratories, Inc., Hayward, Calif.) and water until the experiments. Animals were sacrificed by decapitation and the small intestine was stripped from its mesentery, rinsed with cold saline, and everted sacs (11) of $5 \mathrm{~cm}$ length were prepared with $1.0 \mathrm{ml}$ buffer A without $\left[{ }^{14} \mathrm{C}\right]$ inulin in the interior (serosal) space. Although jejunal and upper ileal sacs were found to have similar hydrolytic and transport properties, sacs prepared from adjacent segments of jejunum were used in comparative studies to minimize variation along the small intestine. 25-ml Erlenmeyer flasks, containing buffer $\mathrm{A}$ and a particular test substrate, were gassed with $95 \% \mathrm{O}_{2} / 5 \% \mathrm{CO}_{2}$ before each experiment, and the sac was then added and the flask capped and gently agitated at 50 times per min in a Dubnoff incubator at $37^{\circ} \mathrm{C}$. The volume of incubation medium varied from 3 to $5 \mathrm{ml}$ depending upon the conditions of the individual experiment but was always sufficient to completely cover the gut sac. At the end of the incubation period, the serosal fluid was drained from the sac and the mucosa recovered by scraping with a glass microscope slide. It was then weighed, homogenized along with the serosal fluid ${ }^{4}$ in a ground glass homogenizer, and deproteinized with $0.5 \mathrm{ml}$ each of $0.3 \mathrm{~N}$ barium hydroxide and $5 \%$ zinc sulfate. After centrifugation at $1,200 \mathrm{~g}$ for $10 \mathrm{~min}, 1.0 \mathrm{ml}$ of supernate was acidified with $0.1 \mathrm{ml} 6 \mathrm{~N} \mathrm{HCl}$. An aliquot $(1.0 \mathrm{ml})$ of incubation medium was treated with $0.1 \mathrm{ml} 6 \mathrm{~N} \mathrm{HCl}$. The mucosal and medium samples were then stored at $-20^{\circ} \mathrm{C}$ for subsequent analysis. Recovery of peptides and amino acids by ion-exchange chromatography or liquid scintillation spectroscopy was $97-100 \%$.

Accumulation of peptidase activity in the incubation medium may occur during in vitro studies. To determine the role of released enzymes in the hydrolysis of the tetrapeptide, the medium was analyzed for activity against a variety of peptide substrates. There were no detectable leucyl- $\beta$-naphthylamide or Gly-Leu-Gly-Gly hydrolase activities and only traces of hydrolytic activity against Gly-Gly and Gly-Pro. However, appreciable activity was found toward Leu-Gly-Gly, amounting to $40 \pm 5 \%$ that of the intact tissue. This activity appeared within a few minutes after the start of incubation and was confined to the soluble fraction of the medium. Cell sap peptidases are known to be inhibited by $0.1 \mathrm{mM} p$-(OH)-mercuribenzoate (12). In preliminary studies, we found that $95 \%$ of the peptidase activity in the incubation medium could be suppressed by PHMB without inhibition of brush border aminopeptidases or transport processes. Hence, $0.1 \mathrm{mM} p$-(OH)-mercuribenzoate was included in the incubation medium of all gut sac experiments.

In vivo perfusion technique. After the rat was anesthetized with $50 \mathrm{mg} / \mathrm{kg}$ pentobarbital, the peritoneal cavity was opened and a small incision made in a segment of jejunum $10 \mathrm{~cm}$ beyond the ligament of Treitz. The tip of a polyethylene catheter ( $3 \mathrm{~mm} \mathrm{ID,} 4 \mathrm{~mm}$ OD) was advanced $2 \mathrm{~cm}$ distally and gently secured with a ligature so that the lumen was occluded. A second cannula was placed similarly at a point $10 \mathrm{~cm}$ distal to the first with the catheter facing proximally. The segment was rinsed with buffer A at $37^{\circ} \mathrm{C}$ to remove food and debris and returned to its original position within the abdominal cavity. The incision was

\footnotetext{
${ }^{4}$ In these short-term incubations, accumulation of substrate or its hydrolytic products was appreciable in the mucosa but usually negligible in the serosal fluid. However, to insure accurate assessment of uptake, mucosal scrapings and serosal fluid were combined for analysis. This constituted the "mucosal sample."
} 
covered with clear plastic wrap and intra-abdominal temperature was maintained at $37^{\circ} \mathrm{C}$ by positioning an incandescent light bulb $30 \mathrm{~cm}$ from the animal. To avoid differential hydrostatic pressures, the two catheter openings were kept on the same horizontal plane; under these conditions, the intestinal loop did not become unduly distended and the outflow rate was identical to the perfusion rate $(0.4$ $\mathrm{ml} / \mathrm{min}$ ). Collections were made into glass tubes heated at $95^{\circ} \mathrm{C}$ for $5 \mathrm{~min}$ and centrifuged at $1,200 \mathrm{~g}$ for $10 \mathrm{~min}$. $1 \mathrm{ml}$ of the supernate was acidified with $0.1 \mathrm{ml} 6 \mathrm{~N}$ $\mathrm{HCl}$ and stored at $-20^{\circ} \mathrm{C}$ for subsequent assay. In some experiments, the perfusion effluent was collected directly into tubes maintained in a dry ice-acetone bath and stored at $-20^{\circ} \mathrm{C}$ for subsequent peptidase assay.

Only preparations free of bleeding that maintained a pink color and active peristalsis were used in these perfusion studies.

Assays. Glucose was measured by Tris-buffered glucose oxidase (13). Peptides and amino acids were analyzed by automated ion-exchange chromatography on a Beckman model 119 instrument (Beckman Instruments, Inc., Fullerton, Calif.) with a $21 \times 0.9-\mathrm{cm}$ column of PA-35 resin. The column temperature was maintained at $56^{\circ} \mathrm{C}$ and elution was accomplished with $0.2 \mathrm{~N} \mathrm{Na}$ citrate buffer, $\mathrm{pH} 4.10$. With this technique, the glycine peak emerged at $28 \mathrm{~min}$, leucine at $39 \mathrm{~min}$, Gly-Gly at $42 \mathrm{~min}$, Gly-Leu-Gly-Gly at $52 \mathrm{~min}$, and Leu-Gly-Gly at $64 \mathrm{~min}$. Since the elution time of GlyPro was very close to that for Leu or Gly-Gly, assays of samples containing Gly-Pro in the test solution were eluted with an initial buffer of $0.35 \mathrm{~N} \mathrm{Na}$ citrate, pH 3.21, followed after $30 \mathrm{~min}$ by a $0.2 \mathrm{~N} \mathrm{Na}$ citrate buffer, pH 4.00. With this program, the glycine peak emerged at $40 \mathrm{~min}$, Gly-Gly at $62 \mathrm{~min}$, leucine at $75 \mathrm{~min}$, Gly-Leu-Gly-Gly at $88 \mathrm{~min}$, and Leu-Gly-Gly at $102 \mathrm{~min}$. The Gly-Pro peak emerged at $73 \mathrm{~min}$ obscuring the leucine peak, but the position of leucine could be advanced to $63 \mathrm{~min}$ and the GlyGly peak retarded to $70 \mathrm{~min}$ by raising the column temperature to $67^{\circ} \mathrm{C}$. In the Gly-Pro inhibition experiments, analysis of samples at the two temperatures produced separate peaks for all fragments of the tetrapeptide.

${ }^{14} \mathrm{C}$-Compounds were analyzed by liquid scintillation spectrometry with use of an external standard for quench correction.

Kinetic experiments. For determination of kinetic constants, five substrate concentrations covering a range above and below $K_{m}$ app were tested in random order in at least six intestinal preparations. Values for maximal rate of disappearance of the peptide $\left(V_{\max }\right)$ and apparent Michaelis constant $\left(K_{m}\right)$ based on initial concentrations of substrate within the medium or lumen were determined from Lineweaver-Burk and Eadie plots (14). Slopes and intercepts were determined by least-square fitting and standard errors of kinetic data were within $10 \%$ of mean values given in the tables.

Calculations. In gut sac studies, rate of disappearance of peptide or appearance of its hydrolytic products in the incubation medium was calculated from the equation:

$$
J_{m}=\frac{\left(C_{1}-C_{2} \cdot \frac{I_{1}}{I_{2}}\right) V_{1}}{A \cdot T},
$$

where $J_{m}=$ rate of substrate disappearance from or appearance in the medium in micromoles per second per square centimeters of intestinal surface area; $C_{1}$ and $C_{2}=$ concentrations of peptide in medium at the beginning and end of the experiment respectively; $I_{1}$ and $I_{2}$ concentrations of the $\left[{ }^{14} \mathrm{C}\right]$ - inulin marker at the beginning and end of the experiment respectively; $T=$ time of incubation in seconds; $A=$ mucosal surface area of the gut in square centimeters and $V_{1}=$ volume of the incubation medium.

The equation used for in vivo perfusion studies was identical except that $C_{1}$ and $C_{2}$ represent concentrations of the perfusate at the infusion and collection sites respectively, and $V_{1}=$ the volume of perfusate.

Rate of uptake of peptide or amino acid in the tissue was calculated from the equation:

$$
J_{t}=\frac{\left(C_{t}-C_{2} \cdot \frac{I_{3}}{I_{2}}-C_{c}\right) V_{t}}{A \cdot T},
$$

where $C_{t}=$ the concentration in the tissue after incubation and $C_{c}$ that in control tissue in which no peptide or other transportable solute was in the incubation medium; $I_{3}$ the concentration of inulin in the tissue after incubation. Other symbols are defined above. Since determination of $I_{3} / I_{2}$ was found in repeated experiments to be $0.14 \pm 0.02$ ( $1 \mathrm{SE}$ ), this value was substituted in calculation of uptake of the ${ }^{14} \mathrm{C}$-labeled substrates. $V_{t}=$ volume of tissue based on its weight with the specific gravity assumed to be 1.0. $J_{t}$ was then expressed as micromoles per second per square centimeters.

In the in vitro studies, filled sacs were observed to have a 6-cm length and a $0.5-\mathrm{cm}$ external diameter. They were considered to represent ideal cylinders (15) of these dimensions with a surface area of $9.4 \mathrm{~cm}^{2}$. In in vivo studies the length of gut segments remained at $10 \mathrm{~cm}$ with a $0.5-\mathrm{cm}$ outside diameter yielding a $15.7-\mathrm{cm}^{2}$ surface area.

\section{RESULTS}

Fate of Gly-Leu-Gly-Gly in gut sac experiments. The incubation of $2.0 \mathrm{mM}$ Gly-Leu-Gly-Gly with gut sacs produced an essentially linear decrease in the concentration of the tetrapeptide over a 30 -min period (Fig. 1). The tetrapeptide appeared to be attacked by sequential removal of an amino acid from its $\mathrm{N}$ terminus since a trace of Leu-Gly-Gly but no Gly-Leu or Gly-Leu-Gly was identified by ion-exchange or thinlayer chromatography. As shown in Fig. 1, the hydrolytic products glycine, leucine and Gly-Gly accumulated rapidly in the medium to $0.3 \mathrm{mM}$ at $5 \mathrm{~min}$. Thereafter, concentrations of the released leucine and Gly-Gly remained constant over the subsequent 15 min, indicating a steady state had been approached with the rate of product uptake by tissue being equal to hydrolysis of the parent peptide. In contrast, free glycine continued to accumulate in the medium throughout the incubation period at rates equal to or greater than those for hydrolysis of the tetrapeptide. After a 20-min incubation, leucine and Gly-Gly concentrations began to increase further in the medium, and additional unidentifiable peaks appeared on peptide-amino acid chromatograms suggesting cessation of transport function and nonspecific diffusion of intracellular substances back into the medium. These findings appeared to reflect tissue damage and 


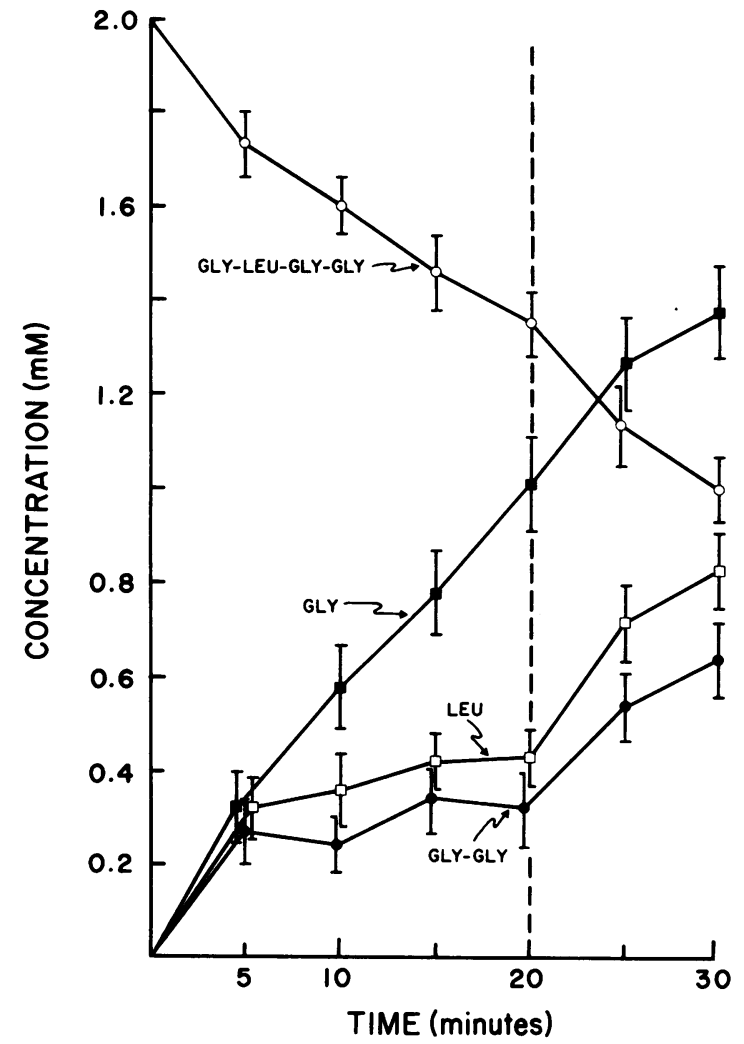

FIGURE 1 Fate of the tetrapeptide, Gly-Leu-Gly-Gly at 2 $\mathrm{mM}$ when incubated with gut sacs in buffer $\mathrm{A}$ as detailed in Methods. The ordinate denotes mean concentrations $( \pm 2 \mathrm{SE}, n=6)$ of the parent tetrapeptide and its products released into the medium. After the initial appearance of final products at $5 \mathrm{~min}$, free glycine continued to accumulate but leucine and Gly-Gly reached a steady state until 20 min incubation. Thereafter, the incremental increase of leucine and Gly-Gly products in the medium was accompanied by appearance of unknown ninhydrin-positive peaks of peptide-amino acid chromatograms suggesting loss of tissue function.

subsequent experiments were carried out for $20 \mathrm{~min}$ or less.

All tetrapeptide that disappeared from the medium could be accounted for by the sum of hydrolytic products in the medium and in the tissue. Table I gives the distribution of the hydrolytic products of Gly-Leu-Gly-Gly after a 10-min incubation with gut sacs and correlates this with maximal rates of hydrolysis or uptake determined from separate kinetic studies with each substrate. Notable are the rapid relative rates of hydrolysis of the parent tetrapeptide and tripeptide as compared to the transport rates of Gly-Gly, leucine and glycine. This explains the appreciable accumulation of the final hydrolytic products in the medium when Gly-Leu-Gly-Gly is the test peptide. Appreciable amounts of the free amino acid products, leucine and glycine, and a trace of Gly-Gly were found in the tissue. That free glycine in the tissue originated solely from Gly-Gly rather than the $\mathrm{N}$ terminal glycine from the tetrapeptide is supported by experiments described below with mixtures of GlyGly and glycine.

Fate of Gly-Gly and glycine. The linear accumulation of free glycine in the medium (Fig. 1) suggested either that this amino acid is not transported under the conditions of the experiments or that appreciable efflux of the amino acid occurs from mucosa after absorption of glycine or Gly-Gly. To determine the uptake of glycine from peptide compared to that from free amino acid, Ringer's solution containing Gly $(2 \mathrm{mM})$ and Gly-Gly $(2 \mathrm{mM})$ with one or the other substrate, uniformly labeled with ${ }^{14} \mathrm{C}$, was incubated with gut sacs. As shown in Table II, tissue glycine was derived completely from GlyGly, there being no net absorption from the free glycine in the medium over the 15 -min period. This finding was reflected in the concentrations of substrate in the medium at the end of the experiment; whereas Gly-Gly concentration decreased from 2 to $1.1 \mathrm{mM}$, there was an increase in free glycine from 2 to 3 $\mathrm{mM}$. Specific radioactivity measurements indicated that the increase in medium glycine was derived entirely from Gly-Gly. Since there was only a trace of Gly-Gly hydrolase activity in the medium at the end of these experiments, the incremental increase in free glycine could have originated from dipeptide that was hydrolyzed either $(a)$ at the cell surface by membrane-bound enzyme or $(b)$ by intracellular peptidases after uptake of intact Gly-Gly. Surface hydrolysis would require that released free glycine be taken up

TABLE I

Gly-Leu-Gly-Gly and Hydrolytic Products in Intestinal Gut Sacs

\begin{tabular}{lccc}
\hline & \multicolumn{2}{c}{ Product distribution* } & \\
\cline { 2 - 3 } & Medium & Tissue & $V_{\max }$ † \\
\hline & $\%$ & $\%$ & \\
Gly-Leu-Gly-Gly & & & 13 \\
Leu-Gly-Gly & Trace & 0 & 20 \\
Gly-Gly & 61 & $39 \S$ & 5 \\
Leucine & 78 & 17 & 3.0 \\
Glycine & 100 & $0 \S$ & 1.0 \\
\hline
\end{tabular}

* Based on a 10-min incubation of $2 \mathrm{mM}$ Gly-Leu-Gly-Gly. Statistical parameters are given in Methods and Fig. 1.

\$ Calculated from separate kinetic experiments with each peptide or amino acid as described in Methods; values given are relative with glycine uptake taken as 1.0. Statistical data for absolute $V_{\max }$ values are given in Table IV.

$\S$ All tissue glycine was derived from Gly-Gly (see Table II) so that no net influx of free glycine occurred when tetrapeptide was the test substrate. 
TABLE II

Uptake of Glycine from Amino Acid and Dipeptide*

\begin{tabular}{cccccc}
\hline & \multicolumn{2}{c}{ Final medium conc. } & \multicolumn{2}{c}{ Intracellular glycine } \\
\cline { 2 - 6 } Medium & Glycine & Gly-Gly & Total & From Gly-Gly & From Glycine \\
\hline & \multicolumn{2}{c}{$m M$} & & \multicolumn{2}{c}{$\mu$ mol/g } \\
Glycine $(2 \mathrm{mM})+$ Gly-Gly $\$(2 \mathrm{mM})$ & $3.0 \pm 0.4$ & $1.1 \pm 0.03$ & $11.4 \pm 1.4$ & $11.2 \pm 0.05$ & - \\
Glycine $\$(2 \mathrm{mM})+$ Gly-Gly $(2 \mathrm{mM})$ & $3.0 \pm 0.04$ & $1.1 \pm 0.07$ & $11.5 \pm 1.8$ & - & 0 \\
\hline
\end{tabular}

* Mean $\pm S E$ for six gut sacs. Incubation with the amino acid-peptide mixture was for 15 min in Ringer's solution (buffer A) as described in Methods. Tissue and medium were analyzed by ion-exchange chromatography and liquid scintillation spectroscopy.

$\$$ Indicates uniformly labeled ${ }^{14} \mathrm{C}$-substrate.

by the gut sac under conditions where uptake of the free amino acid was nil. Hence, it appears that the peptide is transported intact and hydrolyzed intracellularly with some $(\cong 50 \%)$ of the released free glycine subsequently moving back into the medium.

The effect of hypoxia on peptide disappearance. Intestinal transport of glucose, amino acids, and peptides (16) occurs uphill against a concentration gradient by energy-dependent processes but hydrolytic activity of brush border amino-oligopeptidase is energy independent and is not inhibited by a nitrogen atmosphere. ${ }^{5}$ To identify a possible role of these transport mechanisms in the disappearance of Gly-Leu-Gly-Gly, gut sacs were incubated with the peptide under an atmosphere of $100 \% \mathrm{~N}_{2}$. In separate experiments glucose, leucine, and Gly-Gly served as control substrates, each utilizing its own transport process. As expected, hypoxic conditions produced a marked decrease in uptake of glucose, leucine, and Gly-Gly $(P<0.005)$ (Fig. 2). In contrast, tetrapeptide disappearance occurred at the same rates found for oxygenated controls, although uptake of its hydrolytic products leucine and Gly-Gly was markedly inhibited by the hypoxia (Fig. 2, right). No Gly-Leu-Gly-Gly hydrolase activity was present in the medium fluid at the end of the experiment. Taken together, these findings suggest that hydrolysis of the tetrapeptide does not depend on prior transport of the peptide nor does it occur by enzyme free in medium. Instead, the tetrapeptide appears to be hydrolyzed superficially in the tissue at a site proximal to energy utilizing transport processes. The mechanism that can readily account for these findings is hydrolysis at the brush border membrane by the amino-oligopeptidase.

Effect of Gly-Pro on oligopeptide disappearance. Gly-Pro is absorbed intact by the peptide transport mechanism (16) and is presumably hydrolyzed by

\footnotetext{
${ }^{5}$ Smithson, K. W., and G. M. Gray. Unpublished observations.
}

intracellular soluble peptidases (5); it is not a substrate for the brush border peptidases (9). To define the contribution of the peptide transport apparatus in the assimilation of Gly-Leu-Gly-Gly, Gly-Pro was used as a potent inhibitor of the major peptide transport system. As shown in Fig. 3, the dipeptide had only a marginal inhibitory effect on the uptake of glucose or leucine by gut sacs. Although possibly reflecting slight competition for a common energy source, this reduction in uptake was not significantly different from the control group $(P<0.30)$. As expected, Gly-Gly uptake

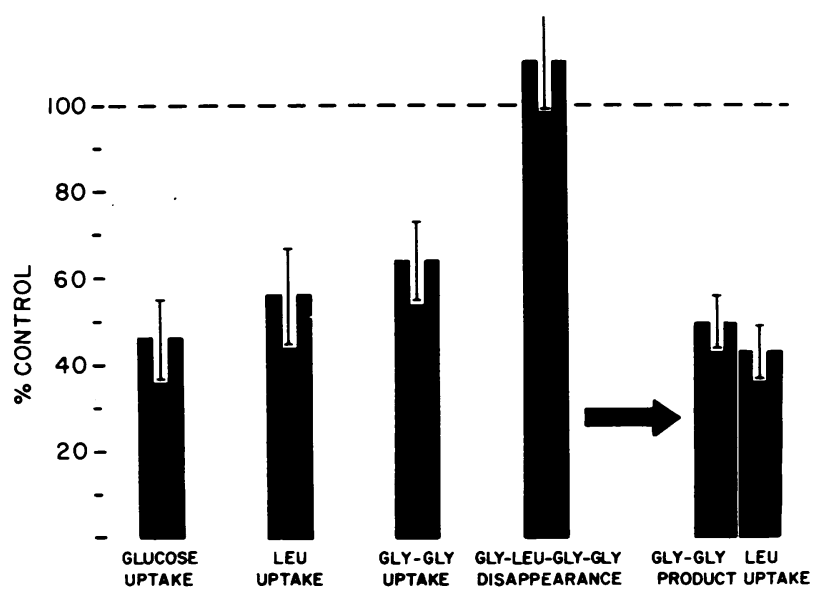

FIGURE 2 Effect of nitrogen atmosphere on the capacity of gut sacs to handle various substrates. The horizontal dashed line constitutes the control sacs incubated in a $95 \% \mathrm{O}_{2}-5 \%$ $\mathrm{CO}_{2}$ atmosphere. Initial concentrations of substrates were 2 $\mathrm{mM}$ except for glucose $(10 \mathrm{mM})$ and uptake or disappearance were determined in 15-min incubations as described in Methods. Each bar represents the mean $\pm 2 \mathrm{SE}$ for six sacs. The arrow points to product uptake from Gly-Leu-Gly-Gly as determined by subtraction of product accumulation in the medium from tetrapeptide disappearance; these values were verified by analysis of total glycine and leucine taken up by the mucosal tissue. Inhibition of uptake of glucose, leucine, and Gly-Gly was appreciable $(P<0.005)$ but no change from the oxygenated control was found for GlyLeu-Gly-Gly $(P>0.20)$. Inhibition of uptake of products of the tetrapeptide was significant $(P<0.005)$. 


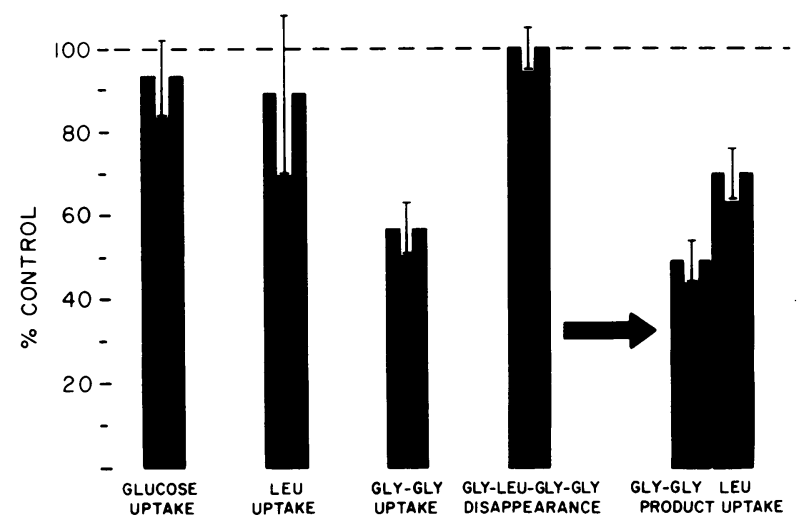

Figure 3 Effect of Gly-Pro ( $10 \mathrm{mM})$ on uptake or disappearance of various substrates by gut sacs as compared to control sacs without Gly-Pro. Conditions were the same as described for Fig. 2. There was no inhibition of Gly-LeuGly-Gly disappearance. The inhibition of uptake as compared to control sacs without Gly-Pro was significant for Gly-Gly $(P<0.001)$ as well as for uptake of the released leucine and Gly-Gly products of the tetrapeptide $(P<0.001)$.

was appreciably inhibited $(P<0.001)$. In contrast, disappearance of Gly-Leu-Gly-Gly continued at the maximal rates observed for control experiments without Gly-Pro suggesting that the tetrapeptide does not utilize the recognized peptide transport mechanism. The uptake of its hydrolytic end-products Gly-Gly and Leu was markedly inhibited $(P<0.001)$ (Fig. 3, right). Inhibition of uptake of the Gly-Gly product was expected and the partial competition for transport between dipeptide and leucine has been observed previously in vitro (16) even though neutral amino acids are considered to be transported independently of Gly-Pro. The inhibition by Gly-Pro of uptake of the released Gly-Gly fragment indicates the absence of a direct coupling of oligopeptide hydrolysis and product transport and suggests that the products are released at intestinal surface membrane at a site functionally proximal to the dipeptide transport mechanism.

Effect of inhibitors of the brush border aminooligopeptidase. In order to study the role of the brush border amino-oligopeptidase on the intestinal assimilation of Gly-Leu-Gly-Gly, the effects of two compounds known to inhibit this enzyme competitively were tested in gut sac experiments. The specific inhibitors were leucyl- $\beta$-naphthylamide (LNA), a synthetic substrate that is hydrolyzed efficiently by the brush border enzyme (9) but not by cell sap enzymes, and the phthalimido derivative of leucine bromomethyl ketone (LPK), a potent aminopeptidase inhibitor (17). Fig. 4 shows the effects of these compounds on the disappearance of glucose, leucine, Gly-Gly, and GlyLeu-Gly-Gly. Glucose and leucine uptake was unaffected indicating absence of nonspecific effects of the inhibitors. Both LNA and LPK reduced uptake of Gly-Gly by $50 \%(P<0.005)$ suggesting that the relatively slow rate of dipeptide transport in vitro may permit some surface hydrolysis by the amino-peptidase in the gut sac. The concept is supported by the in vivo experiments described below. Most notably, both LPK and LNA completely abolished the disappearance of Gly-Leu-Gly-Gly $(P<0.001)$. The complete block in oligopeptide disappearance produced by these inhibitors of the brush border amino-oligopeptidase points to surface hydrolysis as the initial step in the assimilation of the tetrapeptide.

\section{In vivo intestinal perfusion experiments}

Comparison of in vivo and in vitro kinetics. Although in vitro methods such as the gut sac often yield precise information about hydrolysis and transport by intact intestine, such techniques may not provide insight into actual function in vivo, particularly because transport processes may depend upon adequate blood flow. Comparative in vitro and in vivo studies have not previously been carried out for peptides and the hypothetical transport process for oligopeptides such as Gly-Leu-Gly-Gly might not be operative in the in vitro preparation.

Table III compares the fate of Gly-Leu-Gly-Gly in gut sacs and perfused segments of small intestine in vivo. Although the rate of disappearance of the tetrapeptide was no different for the two preparations, hydrolytic products accumulated in the mucosal fluid

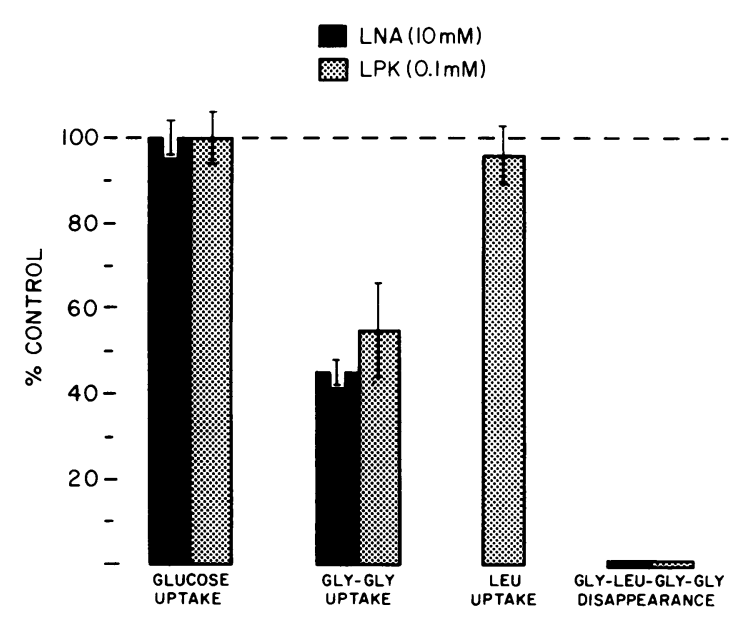

FIGURE 4 Effect of aminopeptidase inhibitors on gut sacs. Leucyl- $\beta$-naphthylamide (LNA) or the phthalimido derivative of leucyl bromomethyl ketone (LPK) were present in the incubation medium at the concentration shown. The \pm 2 SE brackets for Gly-Leu-Gly-Gly disappearance are too small to be shown. Conditions for the experiment are given in Methods and in Fig. 2. There was significant inhibition of Gly-Gly uptake $(P<0.005)$ and Gly-Leu-Gly-Gly disappearance $(P<0.001)$. 
TABLE III

Fate of Gly-Leu-Gly-Gly and Its Hydrolytic Products

\begin{tabular}{lcccc}
\hline & \multicolumn{4}{c}{$10^{4} \times$ Rate } \\
\cline { 2 - 5 } & In vitro & \multicolumn{3}{c}{ In vivo } \\
\hline Tetrapeptide & \multicolumn{4}{c}{$\mu \mathrm{mol} \cdot \mathrm{s}^{-1} \cdot \mathrm{cm}^{-2}$} \\
$\quad$ disappearance & $2.2 \pm 0.2$ & & $2.5 \pm 0.2$ \\
Product accumulation & & & & \\
$\quad$ in medium & & $\%^{*}$ & & $\%^{*}$ \\
Gly & $2.2 \pm 0.3$ & 100 & $0.75 \pm 0.2$ & 30 \\
Leu & $1.7 \pm 0.2$ & 78 & $<0.2$ & $<10$ \\
Gly-Gly & $1.3 \pm 0.2$ & 61 & $<0.2$ & $<10$ \\
\hline
\end{tabular}

In vitro gut sacs and in vivo perfusion of intestinal segments were carried out with $2 \mathrm{mM}$ Gly-Leu-Gly-Gly as described in Methods. Products not found in the bathing medium could be accounted for in the tissue. Values are mean $\pm 2 \mathrm{SE}$ for six experiments.

* Percent represents the proportion of each product released that accumulated in the medium (in vitro) or luminal contents (in vivo).

to a much greater degree in the in vitro system. Notably, only free glycine accumulated in the medium in the in vivo perfusion experiments. These differences suggested either that the tetrapeptide itself is handled differently in vivo or that the released products are transported more efficiently. To distinguish between these possibilities, the tetrapeptide and its potential hydrolytic products were studied individually in the in vitro and in vivo systems and apparent kinetic constants derived, as summarized in Table IV. Notably the $K_{m_{\text {app }}}$ values were similar in vitro and in vivo except for a higher $K_{m}$ for glycine uptake in vivo. In contrast, there was considerable discrepancy between $V_{\max }$ values for transport of glycine, leucine, and Gly-Gly with in vivo uptake rates being 11-30 times more rapid than those found in vitro.
Interestingly enough, the rate of Gly-Leu-Gly-Gly and Leu-Gly-Gly disappearance was nearly identical for in vivo and in vitro experiments. Thus the in vitro gut sac preparation accurately reflects the rates of oligopeptide hydrolysis in vivo but markedly underestimates the true rates of transport processes. Because of the discrepancy between in vitro and in vivo experiments, additional perfusion experiments were undertaken to define the potential role of the brush border amino-oligopeptidase in hydrolysis.

Effect of Gly-Pro, in vivo. As shown in Fig. 5, Gly-Pro produced the expected striking reduction in Gly-Gly uptake in vivo $(P<0.005)$ yet had no significant effect on leucine or glucose transport. As found for the in vitro experiments, there was no inhibition of disappearance of the tetrapeptide, GlyLeu-Gly-Gly, suggesting that this peptide does not share the transport mechanism with Gly-Pro or Gly-Gly.

Effect of inhibition of brush border amino-oligopeptidase in vivo. Analogous to the findings in the gut sac studies, in vivo perfusion of buffer A containing $0.1 \mathrm{mM}$ LPK produced a complete block in tetrapeptide disappearance from the perfusate $(P<0.0005)$ (Fig. 6). Leucine and glucose transport were unaffected and Gly-Gly uptake was reduced only slightly $(-15 \%)$ and this did not quite reach statistical significance $(P>0.05)$. The latter finding differs from the $50 \%$ inhibition by LPK in gut sacs (Fig. 4), probably reflecting the much more efficient function of transport processes in vivo (Table IV). Nevertheless, it seems possible that significant inhibition of GlyGly transport may have been identified by increasing the number of experiments. If so, this might suggest that this dipeptide is hydrolyzed to a minor extent by the brush border amino-oligopeptidase under physiological conditions or that the LPK may have a slight inhibitory effect on intact dipeptide transport. By

TABLE IV

Comparison of Kinetics for Peptides and Amino Acids

\begin{tabular}{|c|c|c|c|c|c|}
\hline & \multicolumn{2}{|r|}{ In vivo } & \multicolumn{2}{|r|}{ In vitro } & \multirow{2}{*}{$\begin{array}{c}V_{\max } \text { ratio } \\
\text { (in vivo/in vitro) }\end{array}$} \\
\hline & $K_{m}^{*}$ & $10^{4} \times V_{\max }$ & $K_{m}$ & $10^{4} \times V_{\max }$ & \\
\hline & $m M$ & $\mu \mathrm{mol} \cdot \mathrm{s}^{-1} \cdot \mathrm{cm}^{-2}$ & $m M$ & $\mu \mathrm{mol} \cdot \mathrm{s}^{-1} \cdot \mathrm{cm}^{-2}$ & \\
\hline Gly-Leu-Gly-Gly & 11 & 17 & 8.3 & 20 & 0.85 \\
\hline Leu-Gly-Gly & 7.0 & 20 & 4.5 & 13 & 1.5 \\
\hline Gly-Gly & 7.0 & 50 & 6.7 & 4.5 & 11 \\
\hline Leucine & 4.0 & 90 & 4.3 & 3.2 & 28 \\
\hline Glycine & 17 & 33 & 2.5 & 1.1 & 30 \\
\hline
\end{tabular}

* $K_{m}=$ apparent Michaelis constant based on observed concentrations in bathing fluid without correction for presence of the unstirred fluid layer over the intestinal surface. Standard errors determined from least-square analysis of kinetic plots were within $10 \%$ of mean values shown. 


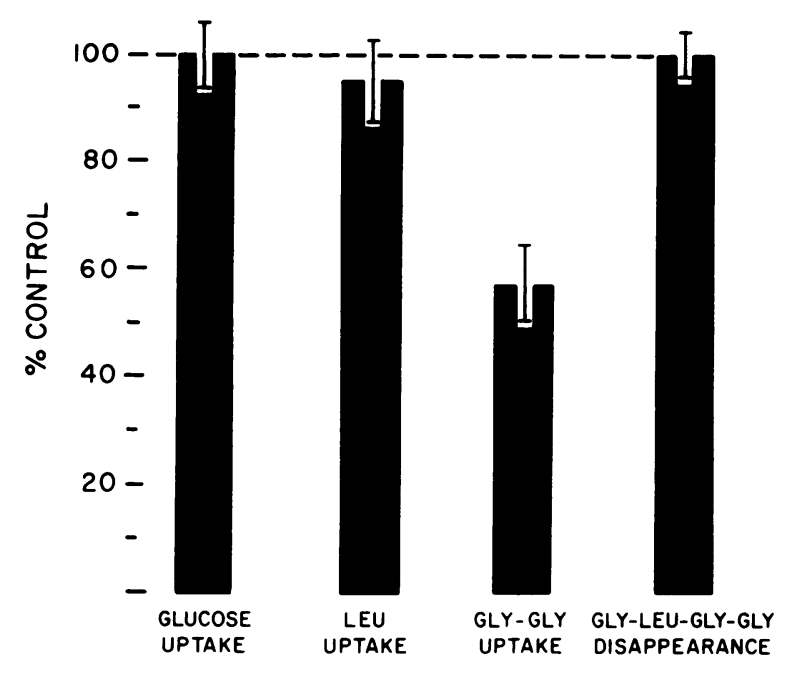

Figure 5 Effect of Gly-Pro $(20 \mathrm{mM})$ on uptake or disappearance of substrate from in vivo jejunal perfusion experiments. The $10-\mathrm{cm}$ length of intestine was perfused at 0.4 $\mathrm{ml} / \mathrm{min}$ with substrate in buffer A as described in Methods. The bars denote mean \pm 2 SE for six animals as compared to control experiments without Gly-Pro. Only trace amounts of products of Gly-Leu-Gly-Gly were found in the collected luminal contents (cf. Table III). There was no inhibition of Gly-Leu-Gly-Gly disappearance but significant reduction of Gly-Gly uptake $(P<0.005)$.

comparison assimilation of Gly-Leu-Gly-Gly appears to be totally dependent on hydrolysis by the brush border amino-oligopeptidase.

\section{DISCUSSION}

Knowledge of the mechanisms whereby the cells of the intestinal mucosa assimilate peptides is important for our understanding of a critical aspect of human nutrition. Yet the processes involved have been difficult to define because available methods do not distinguish clearly between $(a)$ peptide transport followed by intracellular hydrolysis and $(b)$ surface hydrolysis by brush border enzymes followed by transport of released amino acids and smaller peptides. Since the extensive work of Newey and Smyth (2) with GlyGly, investigators have generally stressed the former model and emphasized its key component, transport of intact peptide molecules across the luminal membrane. Rubino et al. (16), using mucosal strips in influx chambers, characterized an energy-dependent transport apparatus for peptides which could be competitively inhibited by dipeptides but not by free amino acids. The extensive work of Matthews in animals (18) and Adibi in man (7) showed a marked advantage for amino acid absorption from small peptides over an equivalent mixture of the free amino acids and recent thorough studies of mutual inhibition between dipeptides (19) have provided additional evidence of a functioning peptide transport system. There seems little doubt that intake peptide transport plays a role in assimilation of many peptides, particularly if the amino acid constituents have compact side chains.

In contrast, indirect evidence from intestinal perfusion experiments in animals (20) and man (7) has suggested that peptides such as Leu-Leu-Leu and LeuGly-Gly may be hydrolyzed to a great extent before absorption since high concentrations of free leucine appear in intestinal fluid simultaneously with the disappearance of the test peptide. This intraluminal accumulation of leucine could be markedly enhanced by perfusion of isoleucine along with the leucinecontaining tripeptide (7) suggesting that the leucine is released at a site where it must compete with isoleucine for the neutral amino acid transport mechanism. Since very little intraluminal peptidase activity was present in these studies, it appeared that hydrolysis of the peptide occurred at the brush border membrane. However, the assimilation of peptides that are not hydrolyzed by brush border enzymes can also be associated with intraluminal accumulation of amino acid products because of back diffusion from an intracellular site of hydrolysis $(2,7)$. Furthermore, the concept that concentrations of hydrolytic products in the bulk phase of intestinal fluid can be kinetically related to their transport rates is no longer tenable because local concentrations of products released by surface digestion within the relatively protected unstirred water layer (15) are likely to be 5-10 times those observed in bulk phase of intestinal fluid. Whether surface hydrolysis followed by transport is associated with high concentrations of products within the mixed intestinal contents will depend on the relative rates of the two processes. For these

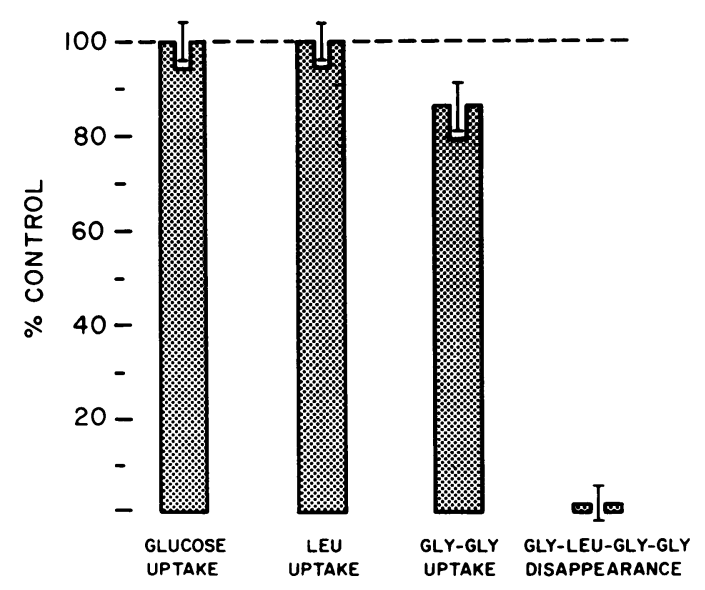

FIGURE 6 Effect of $0.1 \mathrm{mM}$ LPK on uptake or disappearance of test substrates from in vivo jejunal perfusion experiments. Conditions were the same as described in Fig. 5. The apparent reduction of Gly-Gly uptake did not reach statistical significance $(P>0.05<0.10)$. There was a complete block in Gly-Leu-Gly-Gly disappearance $(P<0.001)$. 
reasons, the degree of intraluminal accumulation of hydrolytic products does not constitute valid evidence for either surface hydrolysis or intact transport of a peptide.

In our experiments, a mixed tetrapeptide, Gly-LeuGly-Gly, having one bulky residue was selected to probe the role of the brush border aminopeptidase in peptide assimilation. The pure brush border aminopeptidase possesses high specificity for this peptide (21) $\left(K_{m}=0.5 \mathrm{mM} ; V_{\max }=34 \mu \mathrm{mol} / \mathrm{min}\right.$ per $\mathrm{mg}$ protein). The experiments with gut sacs show that the first stage of intestinal assimilation of this tetrapeptide is independent of an oxygen environment (Fig. 2), is clearly separate from the major peptide transport mechanism (Fig. 3), and yet is completely abolished by competitive inhibitors of the brush border aminopeptidase (Fig. 4). These findings are verified by in vivo perfusion studies (Table III) where maximal rates of tetrapeptide disappearance were comparable to those found in vitro. Since intestinal nutrient transport processes are known to require energy, the continued maximal disappearance of Gly-Leu-Gly-Gly in gut sacs incubated in a nitrogen atmosphere suggests that hydrolysis occurs at the intestinal surface before transport. The appreciable accumulation of its transportable hydrolytic products in the incubation medium in nitrogen experiments (Fig. 2) and the absence of medium peptidase activity are consistent with this interpretation.

The disappearance of Gly-Leu-Gly-Gly might occur by virtue of a previously unrecognized transport mechanism for tetrapeptides. However such a mechanism would be unique in biological systems for several reasons: $(a)$ In contrast to the findings for the oligopeptide transport mechanism in bacteria (22), it would not be inhibited by dipeptides such as GlyPro (Figs. 3, 5), and yet it would be blocked by peptidase inhibitors (Figs. 4, 6) which are dipeptide analogues. (b) Unlike other small intestinal transport systems, the rate of uptake in gut sacs would not be impaired as compared to that found in vivo (Table IV) and most notably $(c)$ the new tetrapeptide transport system would have to be capable of optimal function even in hypoxic environment; notably all known intestinal nutrient transport processes are markedly inhibited by anaerobic conditions. Because of these factors, the intact transport hypothesis does not appear to be tenable for this model tetrapeptide. Instead all of these findings are most compatible with hydrolysis at the intestinal surface, a nonenergy requiring process. Furthermore, the complete block in Gly-LeuGly-Gly assimilation by inhibitors of the brush border aminopeptidase (Figs. 4, 6) provides strong direct support for the concept that tetrapeptide assimilation requires surface hydrolysis by the aminopeptidase.

When considered alone, the gut sac experiments suggest that the hydrolytic rates at the intestinal surface greatly exceed those for transport of the released products (Table I). However, comparison with the in vivo experiments indicates a generalized impairment of transport processes in gut sacs (Table IV). Even though both the half-saturation concentrations $\left(K_{m}\right)$ and maximal rates $\left(V_{\max }\right)$ were comparable for peptide hydrolysis in vitro and in vivo, $V_{\max }$ values for leucine and Gly-Gly uptake were $<10 \%$ of those found in vivo (Table IV). Indeed, free glycine was not taken up by gut sac when equimolar concentrations of Gly-Gly accompanied the amino acid (Table II). These findings were surprising since uptake rates were comparable to those previously observed by others with short-term incubations in vitro (16). This combination of decrease in $V_{\max }$ with preservation of $K_{m}$ in gut sacs suggests there is a reduction in the number of functioning transport sites rather than any qualitative change in the interaction of substrate with the transport binding site. This impairment in vitro may reflect the absence of the intestinal circulatory system. Whatever the mechanism of the transport defect involved, in vitro intestinal preparations appear to take up nutrients suboptimally and caution should be taken when energy-dependent transport is being compared with nonenergy-requiring surface hydrolysis.

Taken together, the in vitro and in vivo experiments indicate a strict dependence on the intestinal surface amino-oligopeptidase for the assimilation of a tetrapeptide that possesses only a single amino acid residue having a bulky side chain. In other studies, we have observed that the specificity of pure rat and human intestinal brush border aminopeptidase depends upon both the number of residues in the peptide and the length of the amino acid side chains $(21,23)$. The presence of three or four residues particularly when there is a bulky side chain at the N-terminus leads to high binding affinity (low $K_{m}$ ) and rapid hydrolytic rates. Hence it seems probable that other peptides of three to four amino acid residues with bulky side chains will also utilize the aminopeptidase. Based on the structure of peptide substrates $(24,25)$, the dimensions of the intact transport apparatus are such that it can optimally handle peptides of two or three residues $(7-11 \AA$ in length) with compact side chains $(<1.0 \AA$ in width). The amino-oligopeptidase would then play a complimentary role by accommodating larger peptides containing three or four residues (11-14 $\AA$ or greater) with prominent side chains

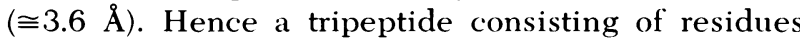
with side-chains of intermediate length $(2.0-3.0 \AA)$ may be handled both by intact transport and surface hydrolysis.

The intestinal stage of peptide assimilation is complex and the brush border amino-oligopeptidase may well play a pivotal role at the lumen-cell interface 
by hydrolyzing the oligopeptides produced from intraluminal pancreatic protease action. The resultant smaller peptides and amino acids could then in turn be transported by known mechanisms. Since intestinal surface digestion may constitute a crucial step in the assimilation of many peptide nutrients, it may be possible to identify patients with intestinal symptoms caused by a genetic defect in the brush border aminooligopeptidase.

\section{ACKNOWLEDGMENTS}

We are grateful to Drs. George I. Glover and John M. Prescott for a gift of the phthalimido derivative of L-leucine bromomethyl ketone.

This work was supported by U. S. Public Health Service National Institutes of Health grant AM 15802.

\section{REFERENCES}

1. Peters, T. J., and M. T. MacMahon. 1970. The absorption of glycine and glycine oligopeptides by the rat. Clin. Sci. (Oxf.). 39: 811-821.

2. Newey, H., and D. H. Smyth. 1962. Cellular mechanisms in intestinal transfer of amino acids. J. Physiol. (Lond.). 164: $527-551$.

3. Adibi, S. A. 1971. Intestinal transport of dipeptides in man: relative importance of hydrolysis and intact absorption. J. Clin. Invest. 50: 2266-2275.

4. Asatoor, A. M., B. Cheng, K. D. G. Edwards, A. F. Lant, D. M. Matthews, M. D. Milne, F. Navab, and A. J. Richards. 1970. Intestinal absorption of two dipeptides in Hartnup disease. Gut. 11: 380-387.

5. Das, M., and A. N. Radhakrishnan. 1973. Glycyl-Lleucine hydrolase, a versatile "master" dipeptidase from monkey small intestine. Biochem. J. 135: 609-615.

6. Matthews, D. M., I. L. Craft, D. M. Geddes, I. J. Wise, and C. W. Hyde. 1968. Absorption of glycine and glycine peptides from the small intestine of the rat. Clin. Sci. (Oxf.). 35: 415-424.

7. Adibi, S. A., E. L. Morse, S. S. Masilamani, and P. M. Amin. 1975. Evidence for two different modes of tripeptide disappearance in human intestine.J. Clin. Invest. 56: 1355-1363.

8. Rhodes, J. B., A. Eichholz, and R. K. Crane. 1967. Studies on the organization of the brush border in intestinal epithelial cells. IV. Aminopeptidase activity in microvillus membranes of hamster intestinal brush borders. Biochim. Biophys. Acta. 135: 959-965.

9. Wojnarowska, F., and G. M. Gray. 1975. Intestinal surface peptide hydrolases: identification and characterization of three enzymes from rat brush border. Biochim. Biophys. Acta. 403: 147-160.
10. Randerath, K. 1968. Thin-Layer Chromatography. Academic Press, Inc., New York. 2nd edition, 110-115.

11. Wilson, T. H., and G. Wiseman. 1954. The use of sacs of everted small intestine for the study of the transference of substances from the mucosal to the serosal surface. J. Physiol. (Lond.). 123: 116-125.

12. Heizer, W. D., R. L. Kerley, and K. J. Isselbacher. 1972. Intestinal peptide hydrolases differences between brush border and cytoplasmic enzymes. Biochim. Biophys. Acta. 264: 450-461.

13. Conklin, K. A., K. M. Yamashiro, and G. M. Gray. 1975. Human intestinal sucrase-isomaltase: identification of free sucrase and isomaltase and cleavage of the hybrid into active distinct subunits. J. Biol. Chem. 250: 5735-5741.

14. Wong, J. T-F. 1975. In Kinetics of Enzyme Mechanisms. Academic Press, Inc., New York. 5-9.

15. Wilson, F. A., and J. M. Dietschy. 1974. The intestinal unstirred layer: its surface area and effect on active transport kinetics. Biochim. Biophys. Acta. 363: 112-126.

16. Rubino, A., M. Field, and H. Shwachman. 1971. Intestinal transport of amino acid residues of dipeptides. J. Biol. Chem. 246: 3542-3548.

17. Kettner, C., G. I. Glover, and J. M. Prescott. 1974. Kinetics of inhibition of aeromonas aminopeptidase by leucine methyl ketone derivatives. Arch. Biochem. Biophys. 165: 739-743.

18. Matthews, D. M. 1975. Intestinal absorption of peptides. Physiol. Rev. 55: 537-608.

19. Das, M., and A. N. Radhakrishnan. 1975. Studies on a wise-spectrum intestinal dipeptide uptake system in the monkey and in the human. Biochem. J. 146: 133-137.

20. Arvanitakis, C., J. Ruhlen, J. Folscroft, and J. B. Rhodes. 1976. Digestion of tripeptides and disaccharides: relationship with brush border hydrolases. Am. J. Physiol. 231: 87-92.

21. Kania, R. K., N. A. Santiago, and G. M. Gray. 1977. Intestinal surface amino-oligopeptidases. II. Substrate kinetics and topography of the active site. J. Biol. Chem. In press.

22. Payne, J. W. 1975. Transport of peptides in microorganisms. In Peptide Transport in Protein Nutrition. D. M. Matthews and J. W. Payne, editors. American Elsevier Publishing Co., Inc., New York. 283-364.

23. Gray, G. M., and N. A. Santiago. 1977. Intestinal surface amino-oligopeptidases. Isolation of two weight isomers and their subunits from rat brush border. $J$. Biol. Chem. In press.

24. Harte, R. A. 1969. Molecules in Three DimensionsA Guide to the Construction of Models of Biochemically Interesting Compounds with CPK Models. American Society of Biological Chemists, Inc., Bethesda, Maryland.

25. Femfert, U., and P. Cichocki. 1974. Hydrophobic areas at the active site of aminopeptidase M. Hoppe-Seyler's Z. Physiol. Chem. 355: 1332-1334. 